\title{
Disrupted functional connectivity of periaqueductal gray subregions in episodic migraine
}

\author{
Zhiye Chen ${ }^{1,2,3}$, Xiaoyan Chen², Mengqi Liư ${ }^{1,3}$, Shuangfeng Liu' ${ }^{1}$, Lin Ma ${ }^{1 *}$ and Shengyuan $\mathrm{Yu}^{2^{*}}$
}

\begin{abstract}
Background: The periaqueductal gray (PAG) dysfunction was recognized in migraine, and the altered dysfunction of PAG subregions were not totally detected up to now. The aim of this study is to investigate the altered functional connectivity of PAG subregions in EM patients.

Methods: The brain structural images and resting state functional MR imaging (rs-fMRI) data were obtained from 18 normal controls (NC) and 18 EM patients on 3.0 T MR system. Seven subregions of PAG were classified as bilateral ventrolateral PAG (vIPAG), lateral PAG (IPAG), dorsolateral PAG (dIPAG) and dorsomedial PAG (dmPAG). The functional connectivity maps of each PAG subregion were calculated, and Two sample $t$-test was applied with age and sex as covariables.

Results: Bilateral vIPAG and left dIPAG presented decreased functional connectivity, and the other subregions (bilateral IPAGs, right dIPAG and dmPAG) showed no significant altered functional connectivity in EM compared with NC. The brain regions with decreased functional connectivity mainly located in bilateral prefrontal cortex(PFC), middle temporal gyrus, primary motor area (PMA) and supplementary motor area (SMA) and right ventrolateral PFC (VIPFC) in EM patients in this study. Disease duration was positively related to the functional connectivity of bilateral VIPAG on the bilateral thalamus and putamen, left pallidum and right medial orbitofrontal gyrus in EM patients.
\end{abstract}

Conclusion: The present study suggested that the dysfunction of bilateral VIPAG and left dIPAG presented in EM, and functional evaluation of PAG subregions may be help for the diagnosis and understanding of EM pathogenesis.

Keywords: Episodic migraine, Functional connectivity, Magnetic resonance imaging, Periaqueductal gray, Restingstate functional MRI

\section{Background}

Periaqueductal gray (PAG) was a center with powerful descending antinociceptive neuronal network $[1,2]$, and the dysfunction in migraine [3] may be considered as a possible "generator" of migraine attacks $[1,4,5]$. Current studies [6,7] supported that PAG network participated in the migraine, which may impair the descending pain modulatory. In episodic migraine (EM) paitents, it had been detected for the nonspecific PAG lesions on T2WI [8], impaired iron homeostasis of PAG [1], and increased

\footnotetext{
*Correspondence: cjr.malin@vip.163.com; yusy1963@126.com

'Department of Radiology, Chinese PLA General Hospital, 28 Fuxing Road, Beijing 100853, China

2Department of Neurology, Chinese PLA General Hospital, 28 Fuxing Road, Beijing 100853, China

Full list of author information is available at the end of the article
}

mean kurtosis and mean diffusivity on diffusion kurtosis imaging [9].

PAG is a small mesencephalic brain structure, and the different subregions may present different specific function. Existing concepts recognized that the anatomical organization of PAG mainly included seven parts: bilateral ventrolateral PAG (vlPAG), bilateral lateral PAG (IPAG), bilateral dorsolateral PAG (dIPAG) and dorsomedial PAG (dmPAG), and PAG connections could also be identified by diffusion tractography and functional connectivity in mammals [10].

The previous document [11] demonstrated that the vlPAG was functionally connected to brain regions associated with descending pain modulation (anterior cingulate cortex (ACC), upper pons/medulla), IPAG and dIPAG 
were connected to brain regions with executive functions (prefrontal cortex, striatum, hippocampus). However, the precise neuromechanism of PAG subregions in the descending pain modulatory system [7] in migraine is not clear.

To investigate the descending pain modulatory circuitry of PAG subregions in migraine, we hypothesize that PAG subregions present different functional connectivity patterns in migraine. To address this hypothesis, we prospectively obtained high resolution structural images and resting state functional MR imaging (rs-fMRI) from 18 EM patients and 18 age- and sex-matched normal controls at first. Secondly, the seed points of PAG subregions were defined, and the functional connectivity was computed. Last, analysis of covariance was performed with age and sex as covariates, and voxel-based correlation was also applied between the functional connectivity of PAG subregions and the clinical variables.

\section{Methods}

\section{Subjects}

Written informed consent was obtained from all participants according to the approval of the ethics committee of the local institutional review board. Eighteen EM patients (15 EM patients without aura and 3 EM patients with aura) were recruited from the International Headache Center, Department of Neurology, Chinese PLA General Hospital from 2014 to 2015. All the following inclusion criteria should be fulfilled: 1) EM is defined as migraine attack days being less than 15 days per month. The definition of migraine refers to 1.1 Migraine without aura and 1.2 Migraine with aura in (ICHD-III beta) [12]; 2) no migraine preventive medication used in the past 3 months; 3 ) absence of any chronic disorders, including hypertension, hypercholesterolemia, diabetes mellitus, cardiovascular diseases, cerebrovascular disorders, neoplastic diseases, infectious diseases, connective tissue diseases, other subtypes of headache, chronic pain other than headache, severe anxiety or depression preceding the onset of headache, psychiatric diseases, etc.; 4) absence of alcohol, nicotine, or other substance abuse. Eighteen NCs were recruited from the hospital's staff and their relatives. Inclusion criteria were similar to those of patients, except for the first two items. NCs should never have had any primary headache disorders or other types of headache in the past year. The exclusion criteria were the following: cranium trauma, illness interfering with central nervous system function, psychotic disorder, and regular use of a psychoactive or hormone medication. The anxiety, depression, and cognitive function of all the participants were evaluated with the Hamilton Anxiety Scale (HAMA) [13], the Hamilton Depression Scale (HAMD) [14], and the Montreal Cognitive Assessment (MoCA) Beijing Version (http://www.mocatest.org).
All the patients were given with the Visual Analogue Scale (VAS) and the Migraine Disability Assessment Scale (MIDAS). MRI scans were taken in the interictal stage at least 3 days after a migraine attack for EM patients. All the subjects were right-handed and underwent conventional MRI examination to exclude the subjects with cerebral infarction, malacia, or occupying lesions. Alcohol, nicotine, caffeine, and other substances were avoided for at least $12 \mathrm{~h}$ before MRI examination.

\section{MRI acquisition}

Images were acquired on a GE 3.0 T MR system (DISCOVERY MR750, GE Healthcare, Milwaukee, WI, USA) and a conventional eight-channel quadrature head coil was used. All subjects were instructed to lie in a supine position, and formed padding was used to limit head movement. Conventional T2weighted image $(\mathrm{TR}=5000 \mathrm{~ms}$, TE $=113.4 \mathrm{~ms}, \quad \mathrm{FOV}=24 \mathrm{~cm} \times 24 \mathrm{~cm}$, Matrix $=384 \times 384)$ and T1-FLAIR $(\mathrm{TR}=2040 \mathrm{~ms}, \mathrm{TE}=6.9 \mathrm{~ms}, \mathrm{FOV}=24 \mathrm{~cm} \times$ $24 \mathrm{~cm}$, Matrix $=384 \times 256)$ were obtained first. Then, the rs-fMRI was performed, during which subjects were instructed to relax, keep their eyes closed, stay awake, remain still, and clear their heads of all thoughts. Functional images were obtained using a gradient echo-planar imaging (EPI) sequence $(\mathrm{TR}=2000 \mathrm{~ms}, \mathrm{TE}=30 \mathrm{~ms}$, flip angle $=90$, slice thickness $=3 \mathrm{~mm}$, slice gap $=1 \mathrm{~mm}$, FOV $=24 \mathrm{~cm} \times 24 \mathrm{~cm}$, Matrix $=64 \times 64)$, and 180 continuous EPI functional volumes were acquired axially over $6 \mathrm{~min}$. Finally, a high resolution three-dimensional T1-weighted fast spoiled gradient recalled echo (3D T1-FSPGR) sequence was performed, which generated 360 contiguous axial slices [TR (repetition time) $=6.3 \mathrm{~ms}$, TE (echo time) $=2.8 \mathrm{~ms}$, flip angle $=15^{\circ}$, FOV (field of view) $=25.6 \mathrm{~cm} \times$ $25.6 \mathrm{~cm}$, Matrix $=256 \times 256$, slice thickness $=1 \mathrm{~mm}$ ]. None of the subjects complained of any discomfort or fell asleep during scanning. No obvious structural damage was observed based on the conventional MR images.

\section{MR image processing}

All MR structural and functional images were processed using Statistical Parametric Mapping 12 (SPM12) (http:// www.fil.ion.ucl.ac.uk/spm) and the rs-fMRI data analysis toolkit (REST v1.8) [15] running under MATLAB 7.6 (The Mathworks, Natick, MA, USA). The data preprocessing was carried out as follows: (1) The first ten volumes of each functional time course was discarded to allow for T1 equilibrium and the participants to adapt; (2) Slice timing; (3) Head motion correction; (4) Spatial normalization. These steps were performed by SPM12. No subjects had head motion with more than $1.5 \mathrm{~mm}$ displacement in $\mathrm{X}, \mathrm{Y}$, and $\mathrm{Z}$ direction or 1.50 of any angular motion throughout the course of the scanning. The linear trend removal and temporal band-pass filtering (0.01$0.08 \mathrm{~Hz}$ ) was performed by REST [15]. 
The functional connectivity analysis was performed as follows: (1) Spatial smooth (full width at half maximum $($ FWHM $)=6 \mathrm{~mm}$ ) using SPM8; (2) The subregions of PAG were classified as seven seeds based on the previous studies [6, 10, 16-19]: 1) left ventrolateral PAG (1_vlPAG)(MNI coordinates: 1_vlPAG $=-4 ;-26 ;-14$ with $2 \mathrm{~mm}$ radius); 2) right ventrolateral PAG(r_vlPAG) (MNI coordinates: $r$ _vlPAG $=4 ;-26 ;-14$ with $2 \mathrm{~mm}$ radius); 3) left lateral PAG(1_lPAG) (MNI coordinates: l_lPAG = $-3 ;-28 ;-10$ with $2 \mathrm{~mm}$ radius); 4) right lateral PAG (r_lPAG) (MNI coordinates: $r$ lPAG $=3 ;-28 ;-10$ with $2 \mathrm{~mm}$ radius); 5) left dorsolateral PAG(1_dlPAG) (MNI coordinates: 1_dlPAG $=-3 ;-30 ;-6$ with $2 \mathrm{~mm}$ radius); 6) right dorsolateral PAG (r_dlPAG) (MNI coordinates: 1_dlPAG $=3 ;-30 ;-6$ with $2 \mathrm{~mm}$ radius); 7) dorsomeidal PAG (dmPAG) (MNI coordinates: dmPAG $=0 ;-30 ;-6$ with $2 \mathrm{~mm}$ radius) (Fig. 1); (3) Functional connectivity computation of the seven subregions of PAG were performed using REST (v1.8). The time courses of subregions of PAG were extracted, and Pearson's correlations were used to calculate the functional connectivity between the extracted time courses and the averaged time courses of the whole brain in a voxel-wise manner. The white matter, cerebrospinal fluid (CSF), and the six head motion parameters were used as covariates. (4) The individual r-maps were normalized to Zmaps using Fisher's Z-transformation.

\section{Statistical analysis}

An independent sample $t$-test was applied to the comparison of the age, HAMA, HAMD and MoCA score between $\mathrm{NC}$ and EM group. Significant difference was set at a $P$ value of $<0.05$. The statistical analysis was performed using SPSS 19.0. Two-sample t-tes was performed to identify the regions with significant differences in connectivity to subregions of PAG, covarying for age and sex, and significance was set at a $P$ value of $<0.001$ without correction. Voxel-based correlation analysis was performed using multiple regression model, and significance was set a $P$ value of $<0.05$ with FDR correction. The minimal number of contiguous voxels was set based on the expected voxels per cluster. The statistical analysis was performed by SPM 12 software.

\section{Results}

Demography and neuropsychological test Eighteen EM patients $(\mathrm{F} / \mathrm{M}=14 / 4)$ and $18 \mathrm{NCs}(\mathrm{F} / \mathrm{M}=14 /$ 4) were enrolled. There was no significant difference for age between EM $(33.39 \pm 10.69$ years old $)$ and CM (39.11 \pm 9.99 years old). There was a significant difference for HAMA between $\mathrm{NC}(9.67 \pm 3.16)$ and EM $(15.67 \pm 9.85)$, HAMD between $\mathrm{NC}(10.89 \pm 7.26)$ and $\operatorname{EM}(15.89 \pm 2.89)$ and MoCA between NC $(27.00 \pm 2.46)$ and EM $(29.17 \pm$ 1.47) $(P<0.05)($ Table 1$)$.

\section{Comparison of the functional connectivity of the subregions of PAG between NC and EM}

Table 2 demonstrated that only right vlPAG, left vlPAG and left dIPAG presented decreased functional connectivity in EM compared with NC, and the other subregions (bilateral IPAGs, right dIPAG and dmPAG) showed no significant altered functional connectivity in EM compared with NC.

The decreased functional connectivity of right vlPAG located in left precentral gyrus (Fig. 2), and the decreased functional connectivity of left vlPAG located in left precentral gyrus, left middle frontal gyrus, left inferior parietal gyrus, bilateral middle temporal gyrus, right superior frontal gyrus and right supplementary motor area (Fig. 3). Figure 4 revealed that the decreased

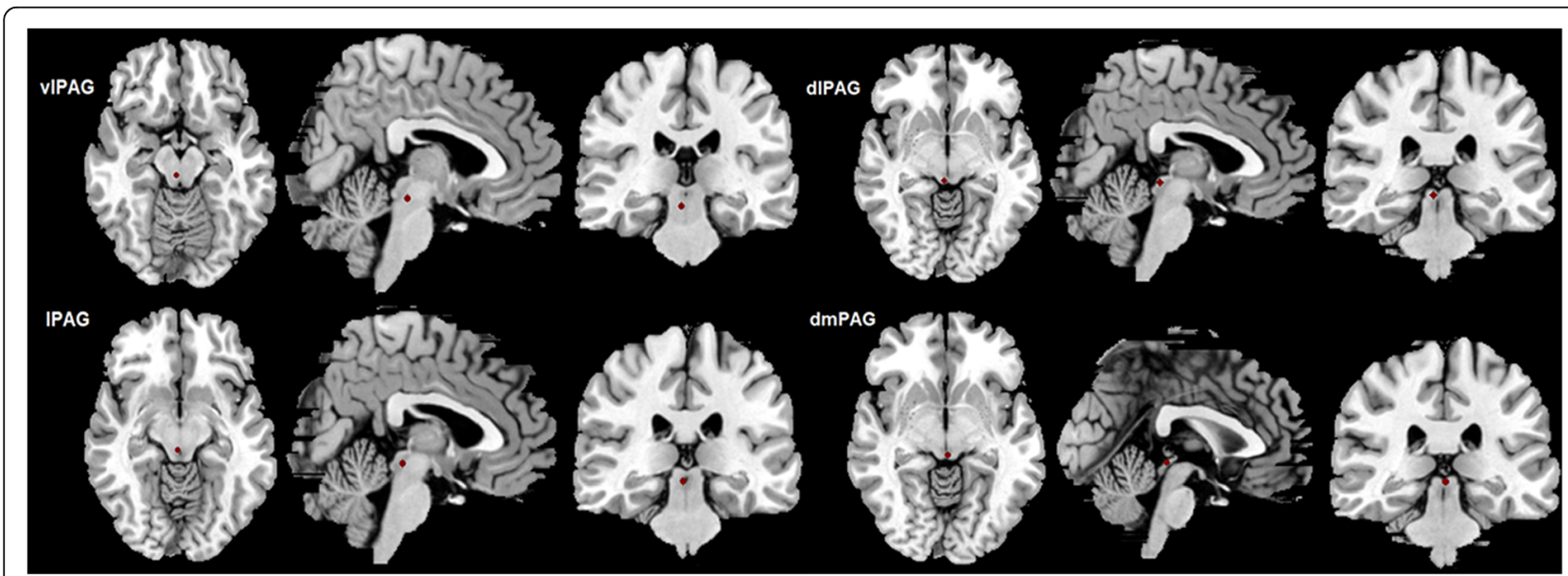

Fig. 1 The seed points of the subregions of PAG. right VIPAG, MNI coordinate:[4-26 -14] with 2 mm radius; right IPAG, MNI coordinate: [3-28 -10] with $2 \mathrm{~mm}$ radius; right dIPAG, MNI coordinate: [3-30 -6] with $2 \mathrm{~mm}$ radius; dmPAG, MNI coordinate: [0-30 -6] with $2 \mathrm{~mm}$ radius 
Table 1 The clinical characteristics of normal controls and EM patients

\begin{tabular}{lll}
\hline & EM & NC \\
\hline Num(F/M) & $18(14 / 4)$ & $18(14 / 4)$ \\
Age & $33.39 \pm 10.69$ & $39.11 \pm 9.99$ \\
HAMA & $15.67 \pm 9.85$ & $9.67 \pm 3.16$ \\
HAMD & $15.89 \pm 2.89$ & $10.89 \pm 7.26$ \\
MoCA & $29.17 \pm 1.47$ & $27.00 \pm 2.46$ \\
DD(yrs) & $12.44 \pm 8.07$ & NA \\
VAS & $8.33 \pm 1.50$ & NA \\
MIDAS & $16 \pm 17.94$ & NA \\
\hline
\end{tabular}

EM episodic migraine, $N C$ normal control, HAMA hamilton anxiety scale, HAMD hamilton depression scale, MoCA montreal cognitive assessment, $D D$ disease duration, VAS visual analogue scale, MIDAS migraine disability assessment questionnaire, $N A$ not available functional connectivity of left dIPAG located in the right parts triangularis of inferior frontal gyrus and medial superior frontal gyrus.

\section{Correlation analysis between the functional connectivity of the bilateral vIPAG and left dIPAG and the clinical variables}

Only disease duration was positively related with the functional connectivity of bilateral vlPAG, and the other clinical variables showed no significant correlation with the functional connectivity of vlPAG (Table 3). Figure 5 demonstrated that the brain regions with positive correlation located in bilateral thalamus and left pallidum between the functional connectivity of left vlPAG and disease duration in EM patients. The brain regions with positive correlation with disease duration located in bilateral thalamus, putamen, and right medial orbitofrontal gyrus for the functional connectivity of right vlPAG

Table 2 The brain regions with altered functional connectivity of PAG over the whole brain between EM and NC

\begin{tabular}{|c|c|c|c|c|c|c|c|c|}
\hline \multirow[t]{2}{*}{ Group } & \multirow[t]{2}{*}{$\mathrm{BA}$} & \multirow[t]{2}{*}{ Anatomic region } & \multicolumn{3}{|c|}{ MNI-space } & \multirow{2}{*}{$\begin{array}{l}\text { Cluster } \\
\text { size }\end{array}$} & \multirow[t]{2}{*}{$P_{\text {uncorr }}$} & \multirow[t]{2}{*}{ Peak T value } \\
\hline & & & $\bar{x}$ & $\bar{Y}$ & Z & & & \\
\hline \multicolumn{9}{|l|}{$\overline{R i g h t \_v I P A G}$} \\
\hline$N C>E M$ & BA6 & Precentral_L & -21 & -15 & 60 & 14 & 0.000 & 4.53 \\
\hline$N C<E M$ & & NA & NA & NA & NA & NA & & NA \\
\hline \multicolumn{9}{|l|}{ Left_vIPAG } \\
\hline \multirow[t]{8}{*}{$N C>E M$} & BA21 & Temporal_Mid_R & 48 & -42 & 3 & 13 & 0.000 & 4.86 \\
\hline & BA6 & Precentral_L & -36 & -9 & 39 & 20 & 0.000 & 4.26 \\
\hline & BA6 & Frontal_Sup_R & 21 & -9 & 66 & 24 & 0.000 & 3.9 \\
\hline & BA6 & Supp_Motor_Area_R & 3 & 12 & 63 & 12 & 0.000 & 4.16 \\
\hline & BA21 & Temporal_Mid_L & -54 & -51 & 9 & 12 & 0.000 & 4.16 \\
\hline & BA46 & Frontal_Sup_R & 27 & 48 & 12 & 15 & 0.000 & 4.14 \\
\hline & BA40 & Parietal_Inf_L & -36 & -39 & 42 & 17 & 0.000 & 4.09 \\
\hline & BA46 & Frontal_Mid_L & -30 & 45 & 21 & 20 & 0.000 & 3.97 \\
\hline $\mathrm{NC}<\mathrm{EM}$ & & NA & NA & NA & NA & NA & & NA \\
\hline \multicolumn{9}{|l|}{ Right_IPAG } \\
\hline$N C>E M$ & & NA & NA & NA & NA & NA & & NA \\
\hline$N C<E M$ & & NA & NA & NA & NA & NA & & NA \\
\hline \multicolumn{9}{|l|}{ Left_IPAG } \\
\hline$N C>E M$ & & NA & NA & NA & NA & NA & & NA \\
\hline$N C<E M$ & & NA & NA & NA & NA & NA & & NA \\
\hline \multicolumn{9}{|l|}{ Right_dIPAG } \\
\hline$N C>E M$ & & NA & NA & NA & NA & NA & & NA \\
\hline $\mathrm{NC}<\mathrm{EM}$ & & NA & NA & NA & NA & NA & & NA \\
\hline \multicolumn{9}{|l|}{ Left_dIPAG } \\
\hline \multirow[t]{2}{*}{$N C>E M$} & BA45 & Frontal_Inf_Tri_R & 48 & 27 & 9 & 12 & & 5.14 \\
\hline & BA8 & Frontal_Sup_Medial_R & 6 & 27 & 57 & 14 & & 5.13 \\
\hline $\mathrm{NC}<\mathrm{EM}$ & & NA & NA & NA & NA & NA & & NA \\
\hline \multicolumn{9}{|l|}{ dmPAG } \\
\hline$N C>E M$ & & NA & NA & NA & NA & NA & & NA \\
\hline $\mathrm{NC}<\mathrm{EM}$ & & NA & NA & NA & NA & NA & & NA \\
\hline
\end{tabular}




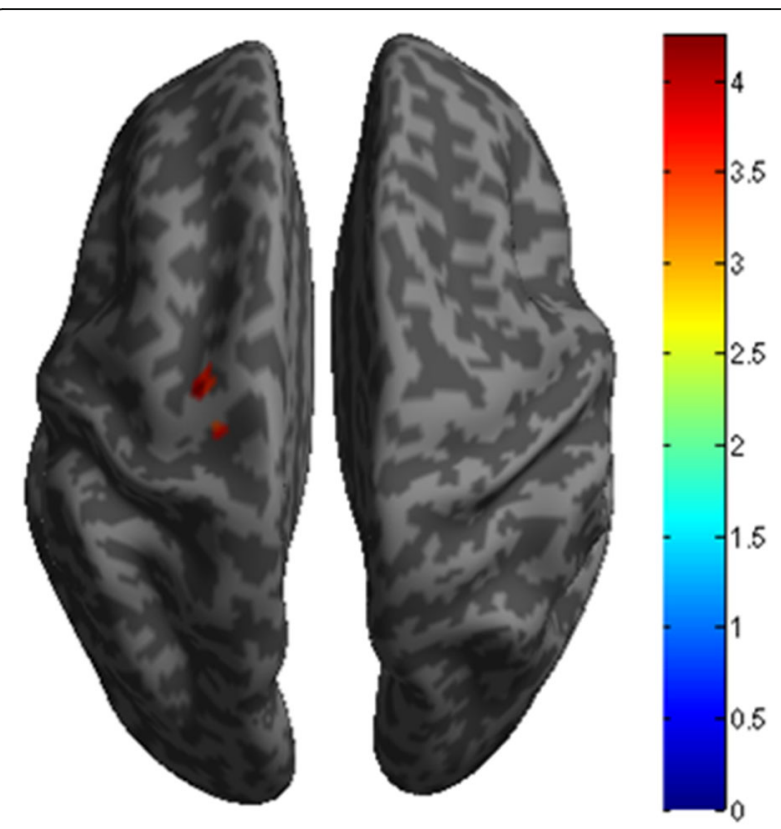

Fig. 2 The decreased functional connectivity of right ventrolateral PAG in EM compared with NC

(Fig. 6). There was no significant correlation between the functional connectivity of left dIPAG and the clinical variables.

\section{Discussion}

This study demonstrated that bilateral vlPAG and left dIPAG may play a role in the descending pain modulatory circuit in EM patients, and the other PAG subregions did not show significant altered functional connectivity in EM. This altered functional connectivity pattern established a concept that PAG subregion analysis may be adapted to the investigation of the pain modulatory in EM while not a whole PAG region analysis. Based on the anatomical organization of PAG, different PAG subregions had different cytoarchitecture and neural connections, therefore, different PAG subregions presented different physiological function [20,21], which may be used to explain the altered functional connectivity of some selected PAG subregions.

In this study, bilateral vlPAG dysfunction were involved with the disrupted functional connectivity in EM patients, especially presented the left predominance. It was known that PAG contains neural circuits that participate in descending antinociception [22], and vlPAG release the antinociceptive peptides Met-enkephalin and neurotensin, which were released when nociception occurred and would result in greater sensitivity of PAG enkephalinergic neurons and greater excitability of the descending PAG output neurons that are responsible for antinociception [23]. The output of vlPAG neurons could also be influenced by GABA recptors, and would disrupt the descending pain control system to spinal dorsal horn in a rat experiments [24]. Furthermore, PAG regions receive the projection from spinothalamic tract via the spinomesencephalic tract to transmit the ascending pain and temperature information [25]. Therefore, vlPAG dysfunction may be considered as an important targeted impaired PAG subregion in EM patients.

In this study, right vlPAG only presented the decreased functional connectivity with left precentral gyrus (BA6, premotor cortex), and left vlPAG showed the decreased functional connectivity with left precentral gyrus, right superior frontal gyrus (BA6, premotor cortex, PMC) and right supplementary motor area (SMA). The decreased functional connectivity between motor cortex and PAG was also demonstrated in fibromyalgia patients [26], which

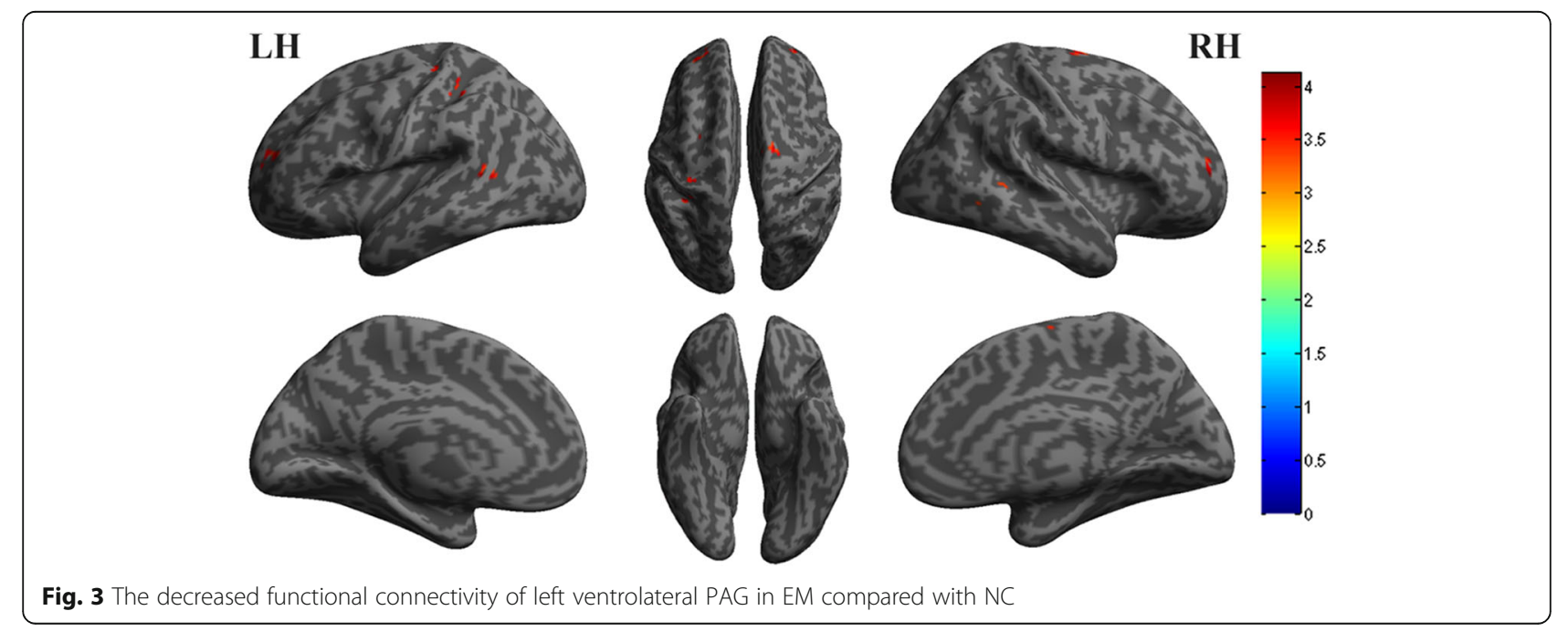




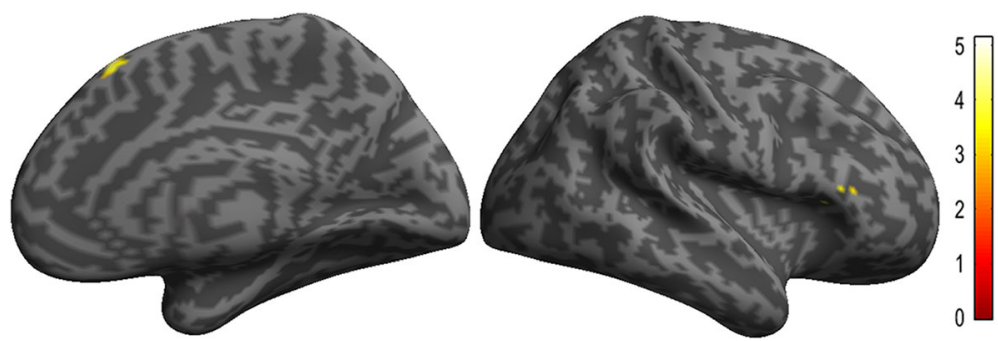

Fig. 4 The decreased functional connectivity of left dorsolateral PAG in EM compared with NC

was also demonstrated in this study and it suggested that vlPAG dysfunction manifested as decreased contralateral functional output from premotor and SMA, and the neuromechanism should further be elucidated.

Left vlPAG presented the decreased functional connectivity with bilateral mid-dorsolateral prefrontal cortex (dlPFC) (BA46) and left dlPAG presented the decreased functional connectivity with right posterior dIPFC (BA8) based on the functional parcellation of PFC. dIPFC mainly lies in the middle frontal gyrus, and it is connected to multiple brain regions, thalamus, basal ganglia and hippocampus, and its function included working memory [27], cognitive flexibility [28], planning [29] and emotion [30]. Although previous document confirmed that PFC dysfunction may be impaired in migraine $[7,31]$, the precise PFC functional location was not involved in these studies,

Table 3 The voxel-based correlation analysis between the functional connectivity of left ventrolateral PAG(vIPAG) and right VIPAG and the clinical variables

\begin{tabular}{|c|c|c|c|c|c|c|c|}
\hline \multirow[t]{2}{*}{ Group } & \multirow[t]{2}{*}{ Anatomic region } & \multirow{2}{*}{$\begin{array}{l}\text { Cluster } \\
\text { size }\end{array}$} & \multicolumn{3}{|c|}{ MNI-space } & \multirow[t]{2}{*}{$P_{\text {uncorr }}$} & \multirow{2}{*}{$\begin{array}{l}\text { Peak } \\
\text { T value }\end{array}$} \\
\hline & & & $\bar{x}$ & $Y$ & Z & & \\
\hline \multicolumn{8}{|c|}{ Left_vIPAG } \\
\hline \multicolumn{8}{|l|}{ DD } \\
\hline \multicolumn{8}{|c|}{ Positive } \\
\hline \multirow{2}{*}{\multicolumn{2}{|c|}{ Thalamus_R }} & 23 & 9 & -3 & 3 & 0.000 & 7.39 \\
\hline & & & 6 & -15 & 6 & 0.000 & 5.38 \\
\hline & Pallidum_L & 15 & -12 & 3 & 0 & 0.000 & 5.8 \\
\hline & Thalamus_L & & -12 & -12 & 0 & 0.000 & 5.68 \\
\hline \multicolumn{8}{|c|}{ Right_vIPAG } \\
\hline \multicolumn{8}{|l|}{ DD } \\
\hline \multicolumn{8}{|c|}{ Positive } \\
\hline & Putamen_L & 104 & -21 & 9 & -9 & 0.000 & 8.75 \\
\hline & Frontal_Med_Orb_R & 12 & 12 & 57 & 0 & 0.000 & 6.31 \\
\hline & Thalamus_L & 14 & -6 & -12 & 3 & 0.000 & 6.06 \\
\hline & Putamen_R & 14 & 24 & 21 & -3 & 0.000 & 5.49 \\
\hline & Thalamus_R & 15 & 6 & -15 & 6 & 0.000 & 5.12 \\
\hline
\end{tabular}

VIPAG ventrolateral PAG, DD disease duration, Positive positive correlation and a recent study demonstrated that migraine without aura showed decreased functional connectivity between PAG and medial PFC (mPFC). In this study, mid-dlPFC and posterior dIPFC presented decreased functional connectivity with left vlPAG and left dlPAG in EM patients, which might be associated with higher HAMA and HAMD scores in EM patients since the dIPFC participated the emotion-nmodulated performance and activity [30]. Therefore, this study suggested that dIPFC might be the targeted impaired brain region in the disrupted vlPAG dysfunction network.

Also, the left dlPAG showed the decreased functional connectivity with right ventrolateral prefrontal cortex (vlPFC) (BA45) in EM patients (Fig. 4). vlPFC was located in the inferior frontal gyrus, and including anterior (BA47), middle (BA45) and posterior (BA44) PFC, which presented different function [32-34]. It was known that right and left vlPFC showed the different specific functions [35], and right vlPFC was thought be play a key role in the motor control [36], and its involvement was not still reported in migraine, and it may be associated with the study methods. In a previous study [37], right anterior vlPFC (BA47) was involved in the integration of emotional processing using a parametric mediation analysis of fMRI. However, right middle (BA45) vlPFC presented decreased functional connectivity with left dIPAG region in the current study. Although it was not consistent with the previous document, it might speculate that middle vlPFC participate in the emotional processing in EM patients since the HAMA and HAMD scores were higher in migraineurs.

Based on two-streams hypothesis [38, 39], dIPFC was the endpoint of the dorsal stream, and vlPFC was the endpoint of the ventral stream. Herein, it could recognize that the endpoints of dorsal and ventral stream were disrupted for the functional connectivity with vlPAG and dIPAG in EM patients, and which may be associated with the visual processing in migraine. In this study, bilateral middle temporal gyrus (BA21) presented decreased functional connectivity with left vlPAG, and BA21 region mainly take part in the processing of visual information, which indicated that disrupted visual information processing may 

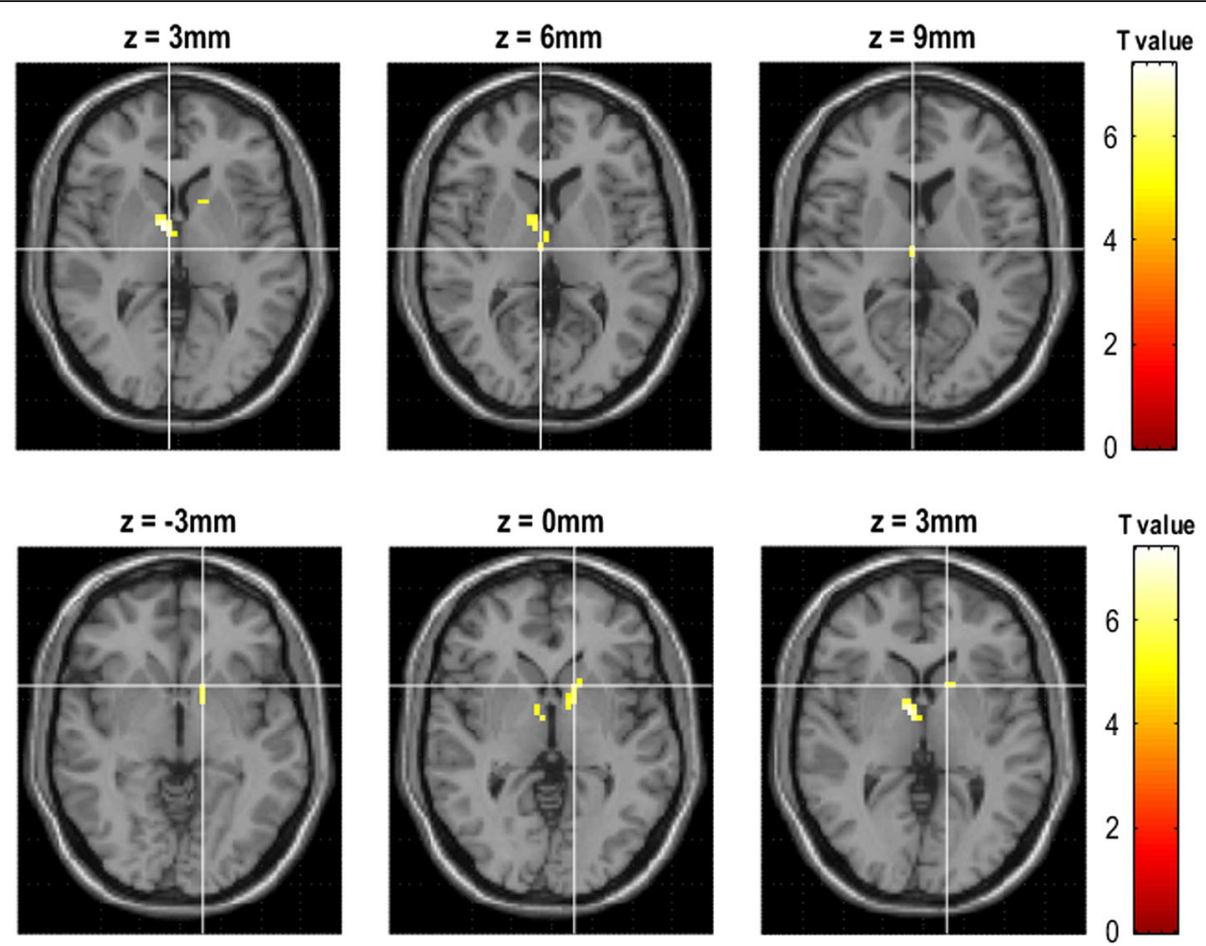

Fig. 5 The brain regions with positive correlation between the functional connectivity of left ventrolateral PAG and disease duration in EM patients

occur in EM patients, and may be associated ventral and dorsal stream.

Voxel-based correlation analysis demonstrated the positive correlation of the diseased duration with the altered functional connectivity of vlPAG, while clinical variables such as VAS, onset frequence, and neuropsychological scale scores were not related with the PAG functional connectivity. These findings indicated that vlPAG dysfunction may be associated with the disease duration, and disease duration may indirectly be used to predict the severity of vlPAG dysfunction in EM patients. A previous study demonstrated that basal ganglia played a significant role in the pathophysiology of the episodic migraine [40], and it also revealed that the disrupted connection with the subcortical gray matter structures (bilateral thalamus and putamen) may be the primary impaired neuromechanism for the descending pain modulation for vlPAG in EM patient.

\section{Conclusions}

In conclusion, disrupted functional connectivity of bilateral vlPAG and left dIPAG presented in bilateral dIPFC, right vlPFC, blilateral PMC and right SMA, bilateral middle temporal gyri in EM patients, and the
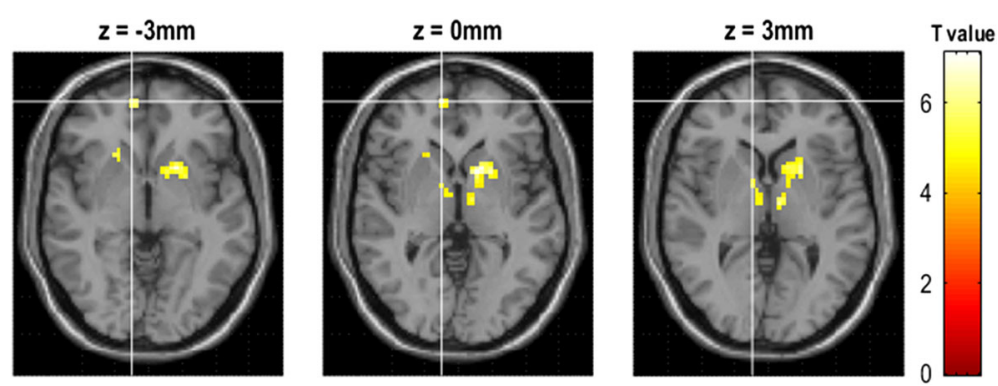

Fig. 6 The brain regions with positive correlation between the functional connectivity of right ventrolateral PAG and disease duration in EM patients 
disease duration was positively related to the functional connectivity of bilateral vlPAG on the bilateral thalamus and putamen, left pallidum and right medial orbitofrontal gyrus in EM patients. These findings suggested that functional evaluation of PAG subregion may be contributed to the diagnosis and understanding of EM pathogenesis.

\section{Abbreviations}

dIPAG: Dorsolateral PAG; dmPAG: Dorsomedial PAG; EM: Episodic migraine; IPAG: Lateral PAG; NC: Normal controls; PAG: Periaqueductal gray; PFC: Prefrontal cortex; PMA: Primary motor area; SMA: Supplementary motor area; vIPAG: Ventrolateral PAG

\section{Acknowledgments}

This work was supported by the National Natural Sciences Foundation of China (81371514), the Special Financial Grant from the China Postdoctoral Science Foundation (2014 T70960) and the Foundation for Medical and health Sci \& Tech innovation Project of Sanya (2016YW37).

\section{Authors' contributions}

Category 1: (a) Conception and Design: LM; SYY. (b) Acquisition of Data: ZYC; MQL; SFL; XYC. (c) Analysis and Interpretation of Data: ZYC. Category 2: (a) Drafting the Article: ZYC. (b) Revising It for Intellectual Content: LM; SYY. All authors read and approved the final manuscript.

\section{Competing interests}

The authors declare that they have no competing interests.

\section{Publisher's Note}

Springer Nature remains neutral with regard to jurisdictional claims in published maps and institutional affiliations.

\section{Author details}

'Department of Radiology, Chinese PLA General Hospital, 28 Fuxing Road, Beijing 100853, China. ${ }^{2}$ Department of Neurology, Chinese PLA General Hospital, 28 Fuxing Road, Beijing 100853, China. ${ }^{3}$ Department of Radiology, Hainan Branch of Chinese PLA General Hospital, Sanya 572013, China.

\section{Received: 1 February 2017 Accepted: 14 March 2017}

Published online: 21 March 2017

\section{References}

1. Welch KM, Nagesh V, Aurora SK, Gelman N (2001) Periaqueductal gray matter dysfunction in migraine: cause or the burden of illness? Headache 41:629-37

2. Smith GST, Savery D, Marden C, Costa JJL, Averill S, Priestley JV, Rattray M (1994) Distribution of messenger RNAs encoding enkephalin, substance $P$, somatostatin, galanin, vasoactive intestinal polypeptide, neuropeptide $Y$, and calcitonin gene-related peptide in the midbrain periaqueductal grey in the rat. J Comp Neurol 350:23-40

3. Raskin NH, Yoshio $H$, Sharon L (1987) Headache may arise from perturbation of brain. Headache 27:416-20

4. Kruit MC, Launer LJ, Overbosch J, van Buchem MA, Ferrari MD (2009) Iron accumulation in deep brain nuclei in migraine: a population-based magnetic resonance imaging study. Cephalalgia 29:351-9

5. Tepper SJ, Lowe MJ, Beall E, Phillips MD, Liu K, Stillman MJ et al (2012) Iron deposition in pain-regulatory nuclei in episodic migraine and chronic daily headache by MRI. Headache 52:236-43

6. Mainero C, Boshyan J, Hadjikhani N (2011) Altered functional MRI restingstate connectivity in periaqueductal gray networks in migraine. Ann Neurol 70:838-45

7. Li Z, Liu M, Lan L, Zeng F, Makris N, Liang Y et al (2016) Altered periaqueductal gray resting state functional connectivity in migraine and the modulation effect of treatment. Sci Rep 6:20298

8. Chen Z, Chen X, Liu M, Liu S, Ma L, Yu S (2016) Nonspecific periaqueducta gray lesions on T2WI in episodic migraine. J Headache Pain 17:101

9. Ito K, Kudo M, Sasaki M, Saito A, Yamashita F, Harada T et al (2016) Detection of changes in the periaqueductal gray matter of patients with episodic migraine using quantitative diffusion kurtosis imaging: preliminary findings. Neuroradiology 58:115-20

10. Menant O, Andersson F, Zelena D, Chaillou E (2016) The benefits of magnetic resonance imaging methods to extend the knowledge of the anatomical organisation of the periaqueductal gray in mammals. J Chem Neuroanat 77:110-20

11. Coulombe MA, Erpelding N, Kucyi A, Davis KD (2016) Intrinsic functional connectivity of periaqueductal gray subregions in humans. Hum Brain Mapp 37:1514-30

12. Headache Classification Committee of the International Headache Society (IHS) (2013) The International Classification of Headache Disorders, 3rd edition (beta version). Cephalalgia 33:629-808

13. Maier W, Buller R, Philipp M, Heuser I (1988) The Hamilton anxiety scale: reliability, validity and sensitivity to change in anxiety and depressive disorders. J Affect Disord 14:61-8

14. Hamilton M (1967) Development of a rating scale for primary depressive illness. Br J Soc Clin Psychol 6:278-96

15. Song XW, Dong ZY, Long XY, Li SF, Zuo XN, Zhu CZ et al (2011) REST: a toolkit for resting-state functional magnetic resonance imaging data processing. PLoS One 6:e25031

16. Yu R, Gollub RL, Spaeth R, Napadow V, Wasan A, Kong J (2014) Disrupted functional connectivity of the periaqueductal gray in chronic low back pain Clin Neuropathol 6:100-8

17. Zyloney CE, Jensen K, Polich G, Loiotile RE, Cheetham A, Laviolette PS et al (2010) Imaging the functional connectivity of the Periaqueductal Gray during genuine and sham electroacupuncture treatment. Mol Pain 6:1-11

18. Menke RA, Jbabdi S, Miller KL, Matthews PM, Zarei M (2010) Connectivitybased segmentation of the substantia nigra in human and its implications in Parkinson's disease. Neuroimage 52:1175-80

19. Kong J, Tu PC, Zyloney C, Su TP (2010) Intrinsic functional connectivity of the periaqueductal gray, a resting fMRI study. Behav Brain Res 211:215-9

20. Hamilton BL (1973) Cytoarchitectural subdivisions of the periaqueductal gray matter in the cat. J Comp Neurol 149:1-27

21. Hamilton BL (1973) Projections of the nuclei of the periaqueductal gray matter in the cat. J Comp Neurol 152:45-57

22. Depaulis A, Keay KA, Bandler R (1994) Quiescence and hyporeactivity evoked by activation of cell bodies in the ventrolateral midbrain periaqueductal gray of the rat. Exp Brain Res 99:75-83

23. Williams FG, Mullet MA, Beitz AJ (1995) Basal release of Met-enkephalin and neurotensin in the ventrolateral periaqueductal gray matter of the rat: a microdialysis study of antinociceptive circuits. Brain Res 690:207-16

24. Yang K, Furue H, Kumamoto E, Dong YX, Yoshimura M (2003) Pre- and postsynaptic inhibition mediated by $\mathrm{GABA}(\mathrm{B})$ receptors in rat ventrolateral periaqueductal gray neurons. Biochem Biophys Res Commun 302:233-7

25. Reddi D, Curran N, Stephens R (2013) An introduction to pain pathways and mechanisms. Br J Hosp Med (Lond) 74(Suppl 12):C188-91

26. Cummiford CM, Nascimento TD, Foerster BR, Clauw DJ, Zubieta JK, Harris RE, Dasilva AF (2016) Changes in resting state functional connectivity after repetitive transcranial direct current stimulation applied to motor cortex in fibromyalgia patients. Arthritis Res Ther 18:1-12

27. Barbey AK, Koenigs M, Grafman J (2013) Dorsolateral prefrontal contributions to human working memory. Cortex 49:1195-205

28. Goldman-Rakic PS (1995) Architecture of the prefrontal cortex and the central executive. Ann N Y Acad Sci 769:71-83

29. Chan RC, Shum D, Toulopoulou T, Chen EY (2008) Assessment of executive functions: review of instruments and identification of critical issues. Arch Clin Neuropsychol 23:201-16

30. Herrington JD, Mohanty A, Koven NS, Fisher JE, Stewart JL, Banich MT et al (2005) Emotion-modulated performance and activity in left dorsolateral prefrontal cortex. Emotion 5:200-7

31. Maniyar FH, Sprenger T, Monteith T, Schankin CJ, Goadsby PJ (2015) The premonitory phase of migraine-what can we learn from it? Headache 55:609-20

32. Gold BT, Balota DA, Jones SJ, Powell DK, Smith CD, Andersen AH (2006) Dissociation of automatic and strategic lexical-semantics: functional magnetic resonance imaging evidence for differing roles of multiple frontotemporal regions. J Neurosci 26:6523-32

33. Badre D, Wagner AD (2007) Left ventrolateral prefrontal cortex and the cognitive control of memory. Neuropsychologia 45:2883-901

34. Danker JF, Gunn P, Anderson JR (2008) A rational account of memory predicts left prefrontal activation during controlled retrieval. Cereb Cortex 18:2674-85 
35. Levy BJ, Wagner AD (2011) Cognitive control and right ventrolateral prefrontal cortex: reflexive reorienting, motor inhibition, and action updating. Ann N Y Acad Sci 1224:40-62

36. Aron AR, Robbins TW, Poldrack RA (2004) Inhibition and the right inferior frontal cortex. Trends Cogn Sci 8:170-7

37. Kida I, Hoshi Y (2016) Right ventrolateral prefrontal cortex involvement in the integration of emotional processing: Parametric mediation analysis of fMRI. Neurosci Lett 615:92-7

38. Mishkin M, Ungerleider LG (1982) Contribution of striate inputs to the visuospatial functions of parieto-preoccipital cortex in monkeys. Behav Brain Res 6:57-77

39. Schenk T, Mcintosh RD (2010) Do we have independent visual streams for perception and action? Cogn Neurosci 1:52-62

40. Maleki N, Becerra L, Nutile L, Pendse G, Brawn J, Bigal M et al (2011) Migraine attacks the basal ganglia. Mol Pain 7:71

\section{Submit your manuscript to a SpringerOpen ${ }^{\circ}$ journal and benefit from:}

- Convenient online submission

- Rigorous peer review

- Immediate publication on acceptance

- Open access: articles freely available online

- High visibility within the field

- Retaining the copyright to your article

Submit your next manuscript at $\gg$ springeropen.com 